INTERNATIONAL DESIGN CONFERENCE - DESIGN 2018

https://doi.org/10.21278/idc.2018.0329

\title{
RESEARCH FOR RELIEF OF DISCOMFORT OF SOUND USER INTERFACE (SUI)
}

\author{
W. Yang
}

\begin{abstract}
Bicycle bells are often used for avoiding danger and can serve as a warning. It seems that psychological displeasure is pronounced when the pedestrian suddenly hears a loud high frequency alarm from the back. In this study, we investigate the conditions of sounds causing little psychological displeasure as a signature needed for interactions informing pedestrians of a bicycle's approach. For this purpose, we analyzed the sounds of existing bicycle horns and investigated factors that concluded to be unpleasant in previous studies and experiments.
\end{abstract}

Keywords: Kansei engineering, interaction design, human behaviour, human centred design

\section{Introduction}

As technology has developed, speakers have become miniaturized and we have entered an era where sound can be easily passed from a small object. However, with the recognition that "it can just ring", sounds that are not properly designed become meaningless and can make people uncomfortable. In fact, due to such sound exhibiting the acoustic characteristics, which can raise more volume than necessary and thus cause anxiety, people who need to be evacuated in emergencies, or hear a departure signal at a train station, can become uncomfortable or enter a state of panic. For example, there have been complaints of rushing flooding into JR (Japan Railway Company).

Professor Iwamiya, sound research expert in Japan was also quoted as saying, "Recently, the necessity of designing an optimum signature tailored to the application has been recognized." In contemporary times where sound is common, we think that designing a signal sound, which plays a signature role, and conveys a specific message while minimizing unpleasantness, is very important.

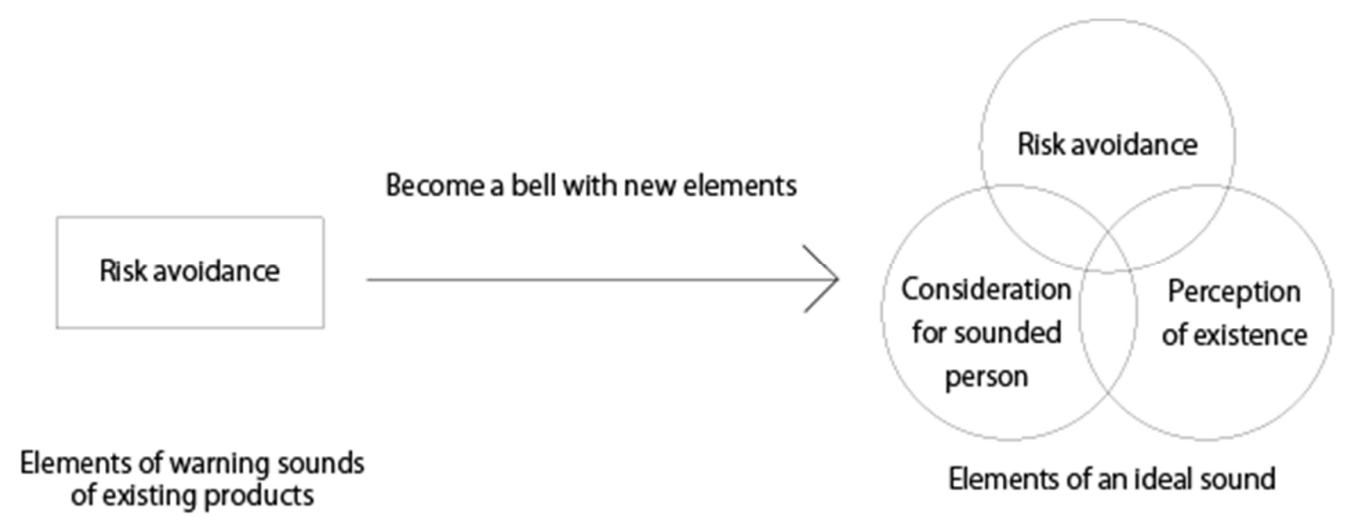

Figure 1. Changes caused by the discomfort of hearing nearby bicycle notification sounds 
In particular, a bicycle bell is often used for both danger avoidance and to issue a warning sound. However, in many cases, these bells are used as approach notification sounds to pedestrians "to notify existence" and to encourage pedestrians to move out of the way in order to prevent accidents before they occur. The pleasant sound of a bicycle bell used as a warning with loud volume and high frequency from behind, thus induces an uncomfortable psychological feeling for pedestrians. For pedestrians and cyclists who often move on the same road, recognition by sound interaction is very important, both to prevent accidents and to create a comfortable road environment for both parties. It is vital that research is carried out on the 'Sound User Interface' in order that warning sounds can be given with the least amount of discomfort (Figure 1).

\section{Research aim}

To identify the conditions that might induce less psychological discomfort for pedestrians when hearing the signature sound from a bicycle bell when used as an approach notification from behind. The purpose of this research is to provide the foundation of research for the new SUI (Sound User Interface) for bicycles.

\subsection{Sound interaction in vehicles}

Depending upon its mobile status, a vehicle may signal to others using sound. This sound is only used when some kind of cooperation system is needed between the vehicle and the recipient of the sound signal, and often uses a sound which incurs as little psychological discomfort for the recipient as possible such that the two parties can interact and cooperate naturally.

\subsubsection{Warning sound in vehicles}

There are many warning sound devices, in a variety of vehicles, which can be used in dangerous situations to prevent accidents. It is important for the person in charge of the vehicle to be able to communicate with the other party in a swift and efficient manner at times of danger. Consequently, the sounds involved are often loud and unpleasant sounds and do not consider the psychological discomfort of others. In addition, it is also important to design suitable notification sounds for vehicles, which can provide information to pedestrians incidentally. It is expected that personal vehicles will diversify in the future, and that the active use of appropriate notification sounds will enhance the appeal of such vehicles. There is little demand for designing sounds for future vehicles based upon the driver. As a foundation for these designs, research is required to identify suitable sounds which will reduce discomfort beyond the basic function of warning sounds.

\subsubsection{The ambiguity of bicycle communication}

The signature sounds of a warning sound device can be adjusted by JIS (Japanese Industrial Standards) to prevent danger. The sound is designed for a crisis situation which will not prevent discomfort. Consequently, pedestrians understand their situation by both hearing the warning sound and by experiencing the discomfort associated with the sound itself.

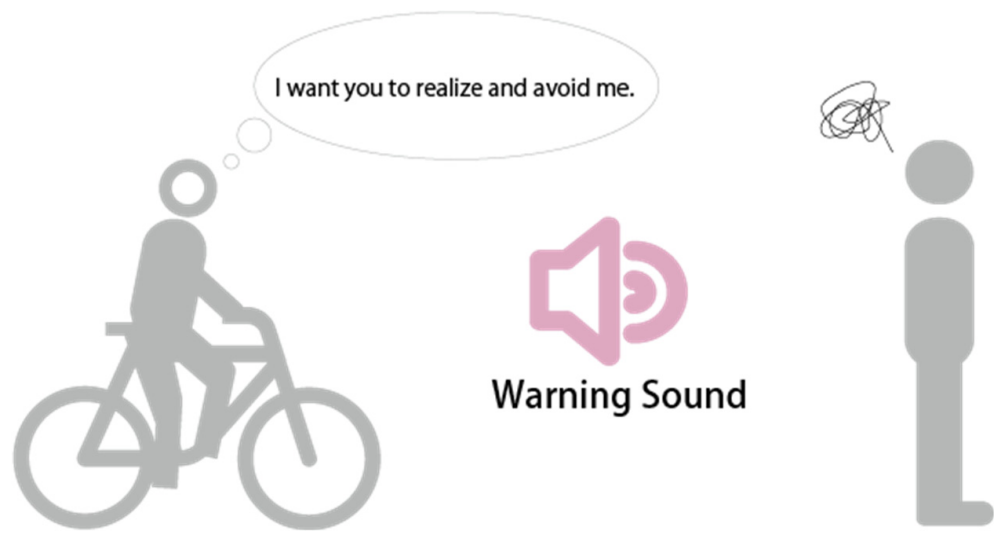

Figure 2. Interaction with a bicycle approach notification sound 
Therefore, it is beginning to be studied to use soft - horn as an approach notification sound even for bicycles. One of the factors is that it has become possible to easily separate and sound multiple sounds by the development of technology (Figure 2).

\section{Research methods and results}

In this research study, we investigated actual movement situations in order to investigate sound conditions suitable for the purpose of approach notifications. Our specific aims are given below:

1. Analyze existing bicycle warning sounds and investigate factors identified in previous research as being unpleasant.

2. Use data to select the condition of a sound which is not felt as unpleasant and create an appropriate sample sound.

3. Use sensitivity evaluation to compare emotions when listening to our sample sound and existing bicycle warning sounds.

4. Use on-road verification equipment to survey sound conditions for practical use.

\subsection{Investigation of elements of discomfort associated with bicycle warning sounds}

We collected the sign sounds from five existing types of warning sound products for bicycles and compared these with the characteristics of sounds which were considered by previous studies to be unpleasant and uncomfortable.

Our market research selected five warning sounds to be studied (Figure 3).

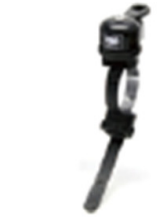

One ringing bell Two ringing bell

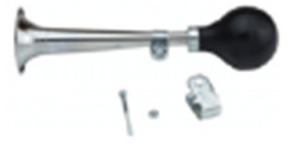

Horn type

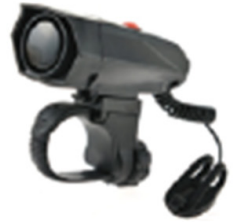

Electronic bell

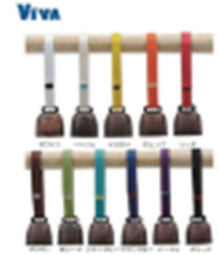

Belling always

Figure 3. Five existing warning sound products for bicycles

\subsubsection{Survey method}

We sampled and investigated the sound of five major products in the following manner.

Recording environment: Indoors within $40 \mathrm{~dB}$

Equipment used: Macbook Pro, Scarlett Studio (Figure 4)

Software: Adobe Audition 2015

Recording method: Sound played once in front of condenser microphone.

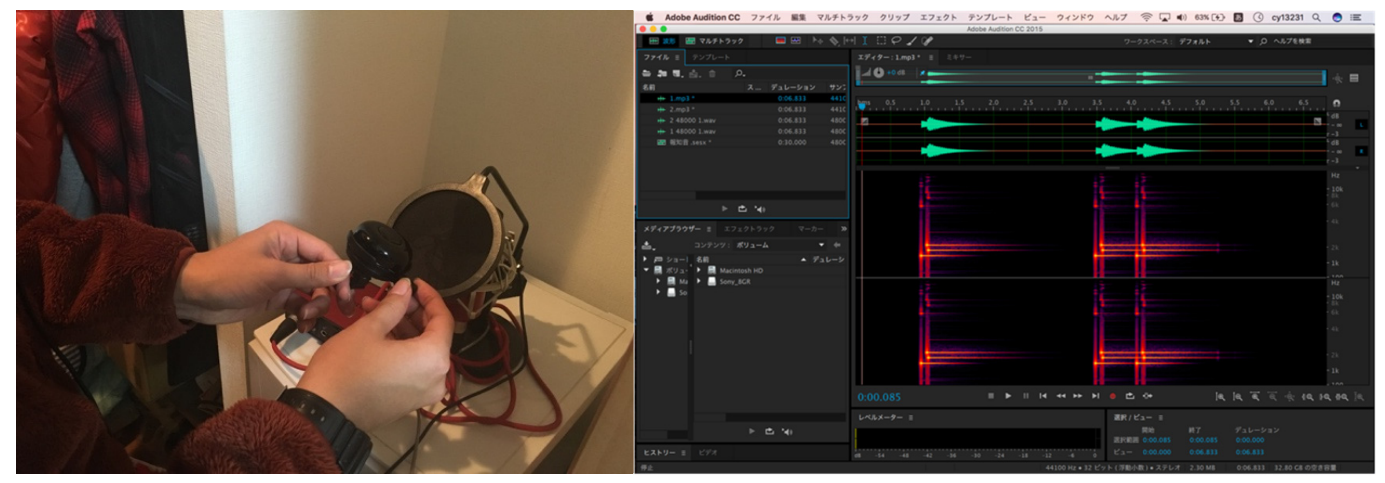

Figure 4. Appearance of sound recording 


\subsubsection{Survey result}

Table 1. Survey result of unpleasant

\begin{tabular}{|r|r|r|r|r|}
\hline & Loudness evaluation & Sound pressure $\mathrm{dB}$ (5 m backward) & Frequency Hz (band 1) & Frequency Hz (band 2) \\
\hline Ideal value & 80 & 80 & 5000 & 7000 \\
\hline One ringing bell & 90 & 84 & 5000 & 13000 \\
\hline Two ringing bell & 90 & 90 & 4000 & 11000 \\
\hline Horn type & 90 & 91 & 1000 & 2000 \\
\hline Electronic bell & Unmeasurable & 99 & $2000-4000$ & $5000-12000$ \\
\hline Belling always & 80 & 81 & 3000 & 12000 \\
\hline
\end{tabular}

Comparing the specific characteristics of unpleasant sounds, we determined that when we emitted the test sound from a point 15 meters behind the recording device, all five existing products exhibited a sound pressure of $80 \mathrm{~dB}$ or more, which was judged to be 'loud'. None of the resultant frequency bands exceeded the value known to cause discomfort $(5,000 \mathrm{~Hz}$ or more) from the first frequency, where the most energy is gathered. However, it was found that it greatly exceeded the value (7,000 Hz or more) showing discomfort to the second frequency (Table 1).

\subsection{Investigation of elements of discomfort associated with bicycle warning sounds}

By carrying out experiments using the multifaceted emotional scale method, we investigated the emotions felt by the sound receivers when hearing warning sounds from the five existing bicycle warning sound products.

\subsubsection{Experimental method}

We recorded environmental sounds at the intersection in front of Tamachi station. Then, we played these environmental sounds from a speaker close to the test subject at approximately $60 \mathrm{~dB}$. We then sounded five types of existing bicycle warning sound devices from $2 \mathrm{~m}$ behind the test subject. After listening to the sound, the subject answered a series of questions on survey form. Then, we examined the emotions felt by the subject with a simplified version of the multifaceted emotional scale method.

Number of subjects: 10 people (Figure 5).
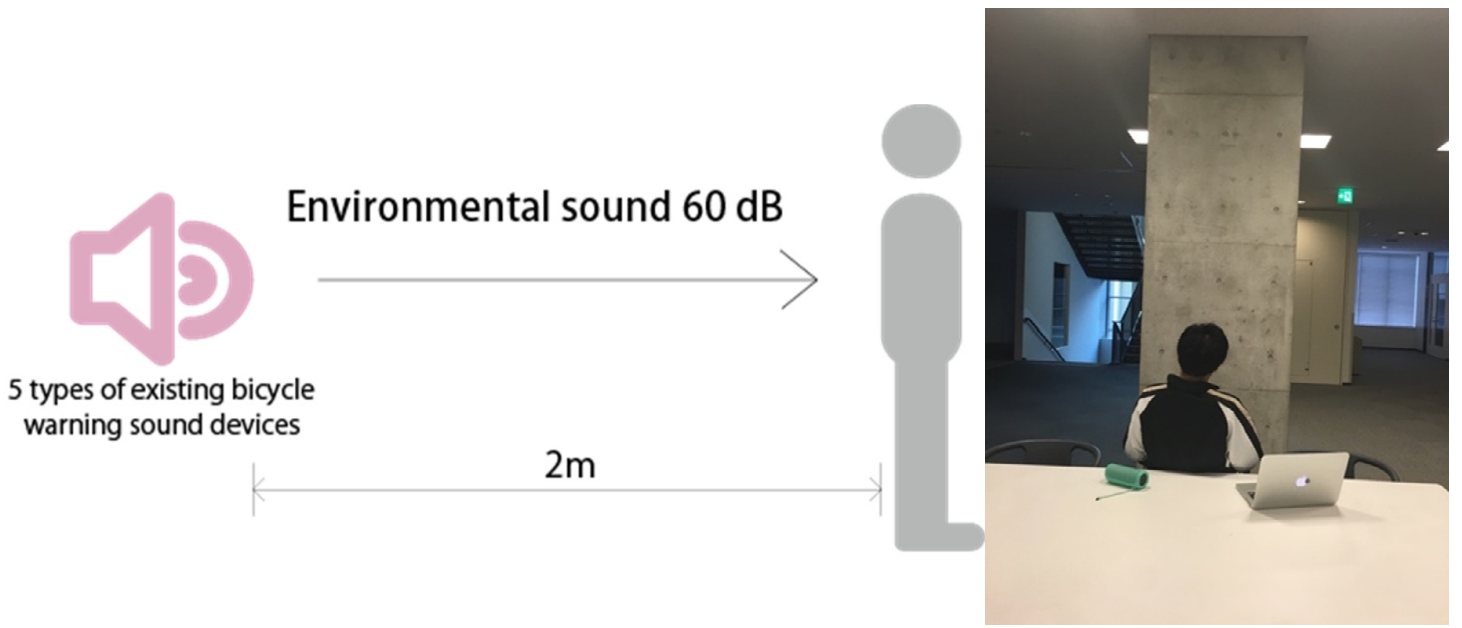

Figure 5. Illustration and appearance of experiment 
Our data identified the following key points:

Table 2. Survey result of unpleasant

\begin{tabular}{|c|c|c|c|c|c|}
\hline & One ringing bell & Two ringing bell & Horn type & Electronic bell & Belling always \\
\hline Depression / anxiety & 80 & n.s & n.s & n.s & 60 \\
\hline hostility & n.s & n.s & n.s & $\mathrm{n} . \mathrm{s}$ & n.s \\
\hline fatigue & n.s & n.s & n.s & n.s & n.s \\
\hline Active comfort & n.s & 50 & n.s & n.s & n.s \\
\hline Inactive comfort & n.s & n.s & n.s & n.s & 60 \\
\hline Affinity & n.s & n.s & n.s & n.s & n.s \\
\hline Concentration & n.s & n.s & n.s & n.s & 50 \\
\hline Startling & 80 & 90 & 80 & 90 & n.s \\
\hline
\end{tabular}

... Emotional state was significant

\section{... Outstanding numerical values compared to others}

1. The subjects indicated that they experienced a high probability of a startled feeling for four of the five existing bicycle warning sound products.

2. There was no commonality in the results in other.

\subsubsection{Considerations}

We can judge a startled experience as being unpleasant because it is felt with 4 kinds other than warning sound equipment with few unpleasant elements. From the above results, the following conditions were considered appropriate for bicycle warning sounds with reduced levels of discomfort.

Sound pressure: $80 \mathrm{~dB}$ or less

- Frequency band 1: less than $5000 \mathrm{~Hz}$

- Frequency band 2: $7000 \mathrm{~Hz}$ or lower

- To suppress emotions of startling

It is difficult to make warning sounds which satisfy such conditions. However, if the notification sound is changed to another sign sound, and such conditions can be satisfied, then the attention of others can still be acquired. In order to make the bicycle approaching notification sound less of a psychologically unpleasant feeling, we considered that it was preferable to design an approaching notification sound instead of a dedicated warning sound.

Based upon previous research, the following conditions were added to the above list of conditions:

- Frequency band 1 is $1500 \mathrm{~Hz}$ or lower $\mathrm{Hz}$

- Frequency band 2: $4000 \mathrm{~Hz}$ or less

- Sound should be applied for 1 to 4 seconds

- Sign should have two sounds with a rising scale

\section{Considerations for alleviating discomfort associated with approach notification sounds}

\subsection{Create an approach notification sound based upon research data}

We created a sample sound based upon our research results, an approaching notification sound for bicycles which met the criteria for an appropriate notification sound. In order to reduce differences due to factors such as existing sign sounds and tone samples were made using the iron harp is the tone sounded the same metal. The production of an approach notification sound focused upon frequency, change in musical scale, and duration of the informing sound. We strived to reduce the difference between existing bicycle earning sounds and other musical expressions and tempos. 


\subsubsection{Production environment}

Music production software: Cubase 8 Element

Input device: Akai Mpk Mini 2 (Figure 6)

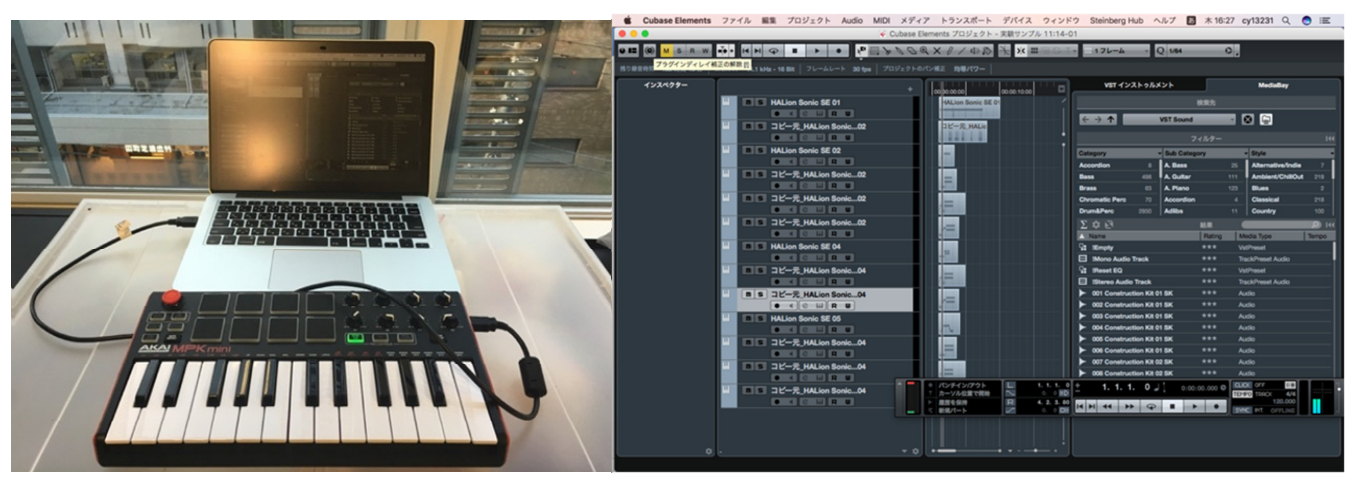

Figure 6. Equipment and production used for the production of a new notification sound

\subsubsection{The signature sound created}

Sound pressure: $78 \mathrm{~dB}$

Frequency band 1: Close to $1000 \mathrm{~Hz}$

Frequency band 2: Approximately $4000 \mathrm{~Hz}$

Change in pitch: $\mathrm{f} 4 \rightarrow$ a 5

Used Instrument: Cubase Preset Instrumental tinkle bell

\subsection{Verification experiment 1}

We then verified that the sample sound created according to the conditions arising from our survey results could reduce the discomfort of the sounded person compared to existing sign sounds.

For this, we used POMS (Profile of Mood States - Brief Form Japanese Version).

POMS is a method for measuring emotional measures and is often used in psychology and psychiatry. Our experimental results yielded the following data:

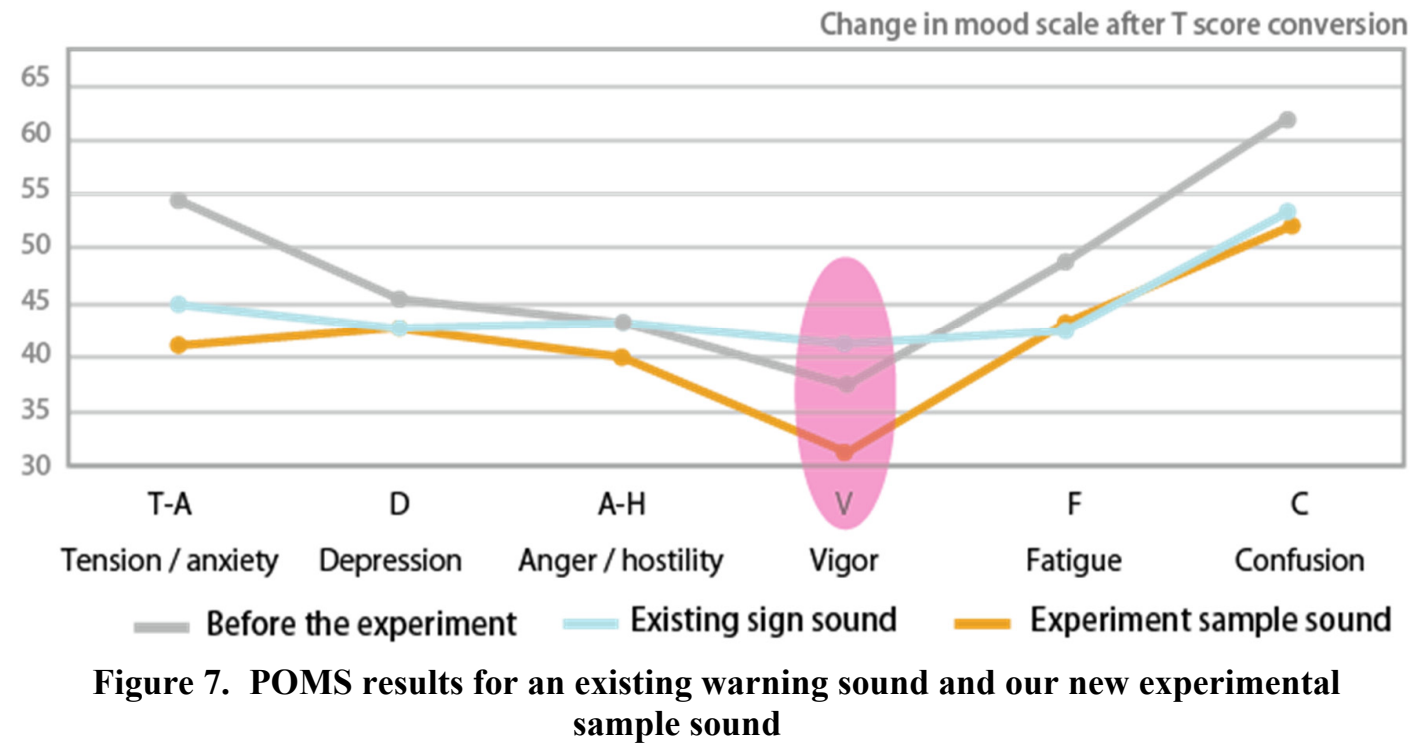

When comparing the results of the existing sign sound and the experimental sample sound, emotion was generally modest. In particular, V (Vigor) showed a difference of more than 10 points between the 
existing signature sound and the experimental sample sound. T-test showed that this numerical difference was significant (Figure 7).

Because $\mathrm{V}$ was higher than before the experiment with the existing sign sound, it appeared that this corresponded to a 'startled' response in the multifaceted emotional scale method. In other words, discomfort can be reduced with sample sounds conforming to the conditions derived from our survey results.

\subsection{Verification experiment 2}

We then performed experiments to compare practical specifications on the street.

To do this, we compared the existing signature sound, the sample sound that we created, and an added melody, in order to confirm the degree of freedom of design.

\subsubsection{Experimental method}

We pedaled a bicycle on a sidewalk at approximately $12 \mathrm{~km} / \mathrm{h}$. We then rang the notification sound 10 meters behind a subject and measured the time from ringing to the point at which the subject turned around. Subjects were then asked to complete the POMS after a series of actions until they gave way. In addition, to reduce the burden on our subjects, we omitted the following POMS criteria as they showed little variation in our laboratory experiments: 'D: depressed-falling', ' $\mathrm{F}$ : fatigue', and ' $\mathrm{C}$ : confusion'.

- Number of Subjects: 13

- We experimented with three kinds of sounds.

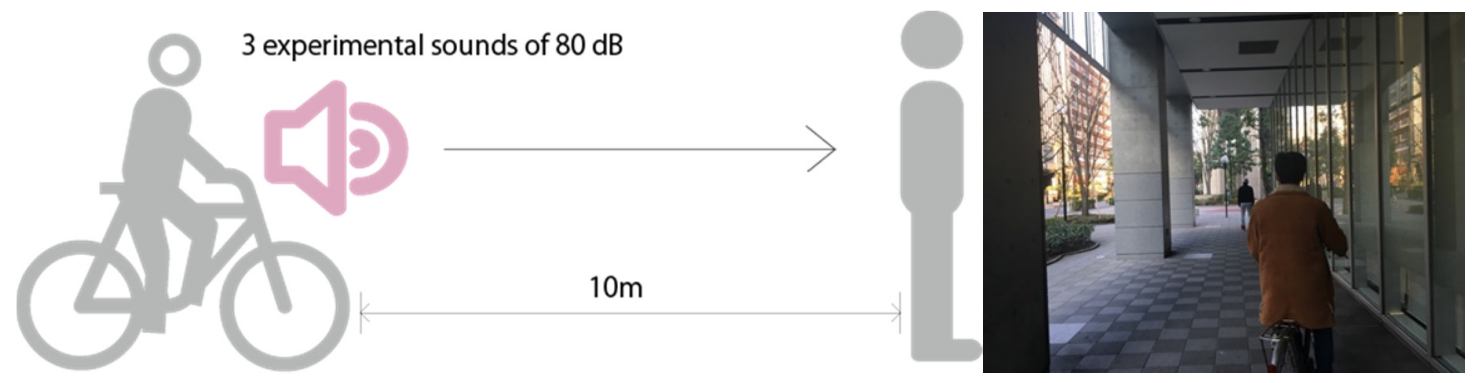

Figure 8. Illustration and appearance of the experiment

Our experimental results revealed the following key findings:

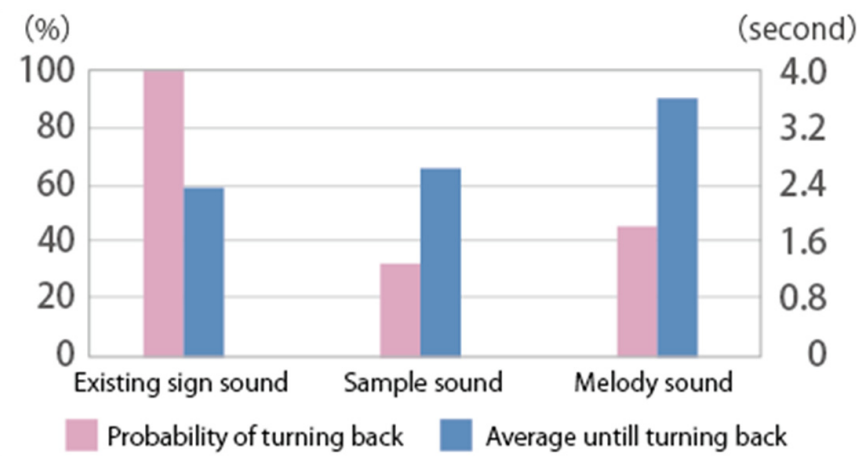

Figure 9. Number of seconds and turning time of each sign sound

Subjects turned away from existing sign sounds and showed reduced reaction speeds. With the sample sound and the melody sound, there were subjects who did not turn around, even if the bicycle approached (Figure 8). According to our interview after the experiment, the reason why subjects did not turn around was as follows: "Because the stereotype of the existing bell sound is strong, I could not detect the bicycle approaching from behind based upon the sounds I heard ". There were many opinions. In addition, there were many opinions relating to the melody sound. For example, "I thought that it was ringing from someone's smartphone" and "It was uncomfortable because I get a cheerful impression from the bicycle approaching" (Figure 9). 
Existing sign sounds and melody sounds did not yield significant results in terms of the three emotional measures tested. On average, the three emotional scales were suppressed (Table 3).

Table 3. Changes in $T$ score and its statistical significance due to differences for Sound 1

\begin{tabular}{|l|c|c|c|c|}
\hline & Existing sign sound & Experiment sample sound & Sound difference & Judgment \\
\hline T-A : Tension / anxiety & 46.7 & 44.1 & 2.6 & $\mathrm{P}<0.1$ \\
\hline A- H: Anger / hostility & 41.1 & 38.8 & 2.2 & $\mathrm{P}<0.1$ \\
\hline V: Vigor & 39.5 & 42.2 & -2.6 & n.s \\
\hline
\end{tabular}

When listening to sounds, the human brain works in a form of concept-driven type of system, which processes sound information according to the expectation and perception of the listener. However, there is a high possibility that subjects judge the approach notification of a bicycle on a road due to discomfort, such as high frequency and large sound pressure (Figure 11, Table 4).

Table 4. Changes in $\mathrm{T}$ score and its statistical significance due to differences for Sound 2

\begin{tabular}{|l|c|c|c|c|}
\hline & Existing sign sound & Melody sound & Sound difference & Judgment \\
\hline T-A : Tension / anxiety & 46.7 & 39.2 & 7.5 & n.s \\
\hline A- H: Anger / hostility & 41.1 & 35.8 & 5.2 & n.s \\
\hline V: Vigor & 39.5 & 36.0 & 3.5 & n.s \\
\hline
\end{tabular}

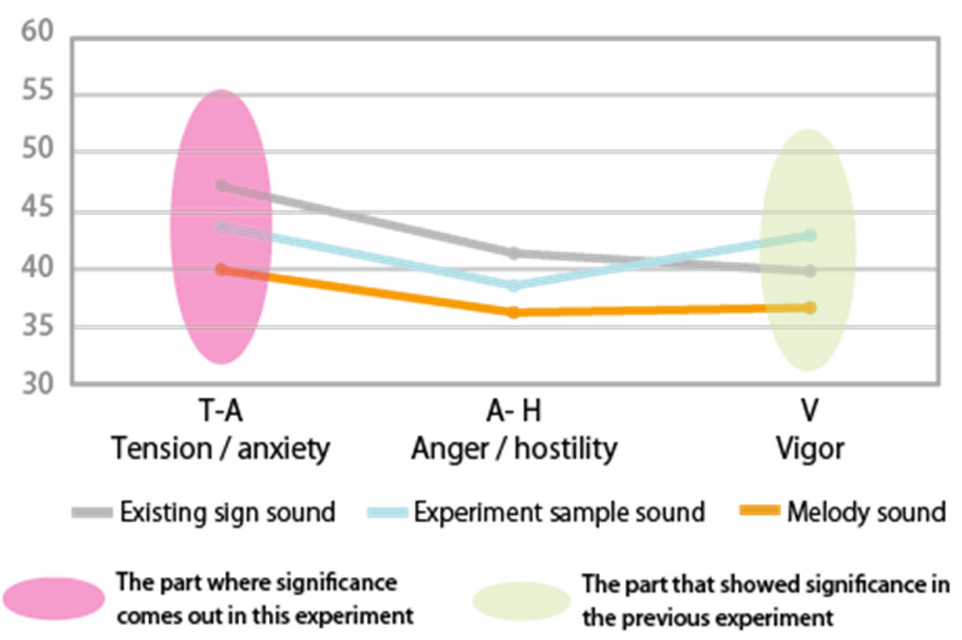

Figure 10. Comparison of $T$ score according to sound differences

However, we consider that some discomfort is also necessary in order to convey the approaching sound and avoid a neglectful response. Furthermore, as the impression given by the melody is strongly biased according to different individuals, this type of sound can be considered as being unsuitable for a bicycle approaching notification sound.

\section{Discussion}

\subsection{Considerations for the practical application of a bicycle approach notification sound with no discomfort}

For the practical application of bicycle approach notification sounds with reduced levels of discomfort, it is necessary to shorten the time from sound recognition to the subject's decision to turn around. 
Significant changes associated with the previous sign sounds which reduced discomfort were accompanied by changes in the judgment of the subject with respect to the approaching notification sound. In order to minimize confusion and judgment, we propose adding the following elements to the practical application of bicycle approach notification sounds with reduced levels of discomfort.

\subsection{Considerations for the practical use of sound}

1. Some discomfort should remain

Completely eliminating discomfort leads to the loss of the important elements related to the bicycle's approach notification sound. In order for subjects to turn around with quick judgment, it is necessary for some discomfort to remain (e.g., large sound pressure, high frequency).

2. Shortening the stimulation time of the sound

It is important to encourage the pedestrian to start judging the sound as early as possible with a short stimulation time. Traditional sound sign research has indicated that notification sounds, using a long stimulation time, are not practical unless pedestrians react quickly. Consequently, a sound stimulation time is required which is shorter than the standard number of seconds used previously.

3. Maintain the acoustic characteristics of a sound hitting metal

A metallic characteristic has been shown to be important in the judgment of sound. Therefore, if we maintain the acoustic characteristics of a notification sound, which sounds like metal hitting metal, judgment should be quicker; for example, the common two-stroke type bell or a onestriking bell, which is heard twice. Nevertheless, it is important to shorten the thinking time for sound by reducing the difference from experience.

4. Avoid adding elements too dissimilar from previous warning sounds

The addition of elements which are too difficult to associate from previous warning sounds, such as the use of complicated melodies, may be misinterpreted by listeners as smartphones, or sounds originating from buildings adjacent to the road. Such signs should be simple in design in order to be effective.

\section{Matters to consider}

Consideration of the practical applications of this research should be based upon the pedestrian's reaction to an approaching notification sound that the pedestrian has not experienced before. Since this is only a consideration for practical use, which matches the current road situation, we consider that the product will change as pedestrians recognize the existence of a bicycle approaching notification sound with reduced discomfort.

However, we consider that retaining some level of discomfort and shortening the sound stimulus should be a common condition, even after cognition. We consider that reducing the reaction time to sound, and differentiating it from other sound information by frequency and volume, is an indispensable element for developing new bicycle approach notification sounds.

\section{Conclusion}

We examined the conditions of sounds exhibiting little psychological discomfort as potential sign sounds for a bicycle to provide pedestrians with an approach notification. As a result of this research, the following items were identified as elements of discomfort in relation to bicycle warning sounds.

- A large sound pressure of $80 \mathrm{~dB}$ or more

- A Second High frequency band in excess of $7000 \mathrm{~Hz}$

When used as an ordinary warning sound, these acoustic characteristics cause no problems because they notify a second party of a potential crisis situation very quickly due to the high discomfort of the sound itself. However, we consider that such discomfort is too excessive for use as an approach notification. In order to use a sound for approach notification, it is necessary to reduce the level of discomfort. As a result of our research, we identified that the following conditions are suitable for a bicycle approach notification sound, which exhibits a lower level of discomfort for use as a close notification.

- Frequency band 1: less than $5000 \mathrm{~Hz}$ 
- Frequency band 2: $7000 \mathrm{~Hz}$ or lower

- Sound stimulation time: 4 seconds or less (Shorter the better)

- Having two notes with a rising musical scale (a complete 5 degree rise)

- Sound pressure level within $80 \mathrm{~dB}$

However, it cannot be said that an approach notification sound with reduced levels of discomfort is a suitable sound for a bicycle approach notification. Previous warning sounds were able to bring about a reaction that causes a pedestrian to turn quickly because the pedestrian clearly understood the level of discomfort and the meaning of this sound on the road because of prior experience. In contrast, an unexperienced pedestrian does not delay, or turn around, in response to an approaching notification sound with reduced levels of discomfort.

In order to create approach notification sounds with reduced levels of discomfort for practical use, in addition to conditions for reducing discomfort, it is necessary to include conditions which illicit reactions from pedestrians, such as turning around quickly. After considering these two conditions, we consider that the conditions required must take into consideration a balance between the reduction of discomfort and the response speed of pedestrians.

\section{References}

Inoue, M. and Hirahara, T. (2009), IEICE Tehnical Report 109(240): The research domain and scale construction of adjective-pairs in a semantic differential method in Japan, , The Institute of Electronics, Information and Communication Engineers.

Iwamiya, S. (2012), Science of sign sound, Corona.

Nishida, K. and Shinjo, I. (2000), Sharp Technical Report No. 77: Sounds of Nifty Advice Reaching Senior Consumers, Sharp.

Sawada, Y. and Hirahara, T. (2009), IEICE Technical Report 109(240): Spectral features that cause unpleasantness of absolutely unpleasant sound, The Institute of Electronics, Information and Communication Engineers.

Tsuji, S. (1997), Science of Kansei - Approach to Kansei Information Processing, Saiensu Publishing.

Wake, S. (2013), "Sound Image of Sound Sign Which Uses Pitch Interval”, Noise Control Engineering Journal, Vol. 37 No. 4, pp. 201-205.

Yang, W.-S. (2013), “A Suggestion to Support the Communication Activities of the Elderly", Proceedings of the 5th International Congress of the International Association of Societies of Design Research (IASDR 2013), Tokyo, Japan, August 26-30, 2013.

Yang, W.-S., Kondo, H. and Seok W.-M. (2015), “Operation Sound Influence on Table Device Character Input Operation”, Proceedings of HCI International 2015 - Posters' Extended Abstracts, Los Angeles, CA, USA, August 2-7, 2015, pp. 51-56.

Wonseok Yang, Associate Professor

Shibaura Institute of Technology, Engineering and Design

Minato-Ku, Shibaura 3-9-14, 108-8548 Tokyo, Japan

Email: yang@shibaura-it.ac.jp 